\title{
THE RESPONSES TO PAINFUL STIMULI OF PATIENTS WITH SEVERE CHRONIC PAINFUL CONDITIONS
}

\author{
BY MARGARET A. KENNARD 1 \\ (From the Department of Surgery, University of Oregon Medical School, Portland, Oregon)
}

(Submitted for publication March 1, 1951; accepted December 10, 1951)

In the study of patients who have severe and prolonged, chronic, painful conditions, it is a clinical fact that some will react more profoundly than others to what are apparently similar conditions. It is also obvious clinically that pain is an extreme fear- and anxiety-provoking stimulus. Furthermore, the diagnosis of "functional pain," or of a "functional element" in pain, is one frequently attached to many of these patients. Because of the confusion generated by these clinical observations it was thought possible that a study of the responses of such patients to painful stimuli might aid in their diagnosis and perhaps add to the knowledge of the physiology of pain.

Such an investigation has been made possible by the recent studies on the physiology of pain which have resulted in a better definition of this very difficult subject. Wolff and his associates have demonstrated that a pain threshold can be quantitatively measured (1); that the perception of pain intensities is relatively fixed for a given person under given environmental conditions; and that thresholds for pain perception vary relatively little from person to person (2). They have also described reaction to pain which is a more variable factor and is observable as a somatic response on the part of the patient. This reaction is related, in their opinion, to bodily and emotional phenomena such as fear, anxiety or apprehension which are widely variable in different people and from time to time in the same person (3).

The present study is an attempt to compare the thresholds and pain reactions of normal subjects with those of patients who have been undergoing severe pain. In this study a third factor has also been examined, namely, the conditioning time to painful stimuli. This part of the project is based on the studies of Welch and Kubis (4) who demonstrated that the conditioning time of neurotic

\footnotetext{
1 Present address: Department of Neurological Research, University of British Columbia, Vancouver, Canada.
}

patients with severe anxiety was significantly shorter than that of normal controls.

\section{METHOD}

Pain threshold: An electrical stimulus was chosen for the measurement of pain threshold. This was standardized in the Laboratory by tests carried out on staff members prior to the present investigation. A Goodwin Stimulator (model 3) was used which was so set as to deliver a single shock of uniform wave-form (20 sigma falling phase) and of varying voltage intensity. The stimulus strength was always increased in 10-volt units, a gradient which made single unit changes barely perceptible. The stimulus was delivercd to the thin-skinned area of the volar surface of the upper left forearm. Contact was made through two small solder bars about $1 \mathrm{~cm}$. long and $3 \mathrm{~mm}$. wide which were fastened parallel and about $3 \mathrm{~mm}$. apart onto a rubber base. The same pair of electrodes was used for all subjects in the present tests. Electrodes were held in place by a rubber strap around the arm such as is used for EKG electrodes. The skin beneath the electrodes had been previously rubbed vigorously with a small amount of electrode jelly.

Galvanic skin response: The reaction to pain was recorded by means of the galvanic skin response (GSR). This reaction is known to result from a sudden change in sweating and to be dependent on arousal or startle in the subject. It was recorded through electrodes attached to the right wrist and palm. One solder electrode, about $11 / 2 \mathrm{~cm}$. in diameter, was pressed against the right palm and a second, about $1 / 2 \mathrm{~cm}$. in diameter, against the volar surface of the right wrist. Each electrode was fastened to a rubber base which was round and about $4 \mathrm{~cm}$. in diameter, held against the skin by a strap. Contact was made by rubbing the skin with electrode paste and then covering each electrode with the jelly. If the contact became dry during the procedure, artefacts appeared in the record and additional jelly was inserted between the electrode and the skin. A ground electrode was fastened to the right ear. The GSR was recorded on an eight-channel Grass Electroencephalograph according to the method described by Redlich (5). The machine was run at "slow" speed, i.e., $11 / 2 \mathrm{~cm} . / \mathrm{sec}$.

Conditioning response: Subjects were conditioned to either painful or auditory stimuli according to the procedure described by Welch and Kubis (4). In order to be sure of comparable findings a duplicate of their film-sequence of nonsense syllables of low association value was obtained from Dr. Welch and his procedure was followed as precisely as possible. 
Subjects were asked to read out loud a series of nonsense syllables projected before them, one every six seconds. The unconditioned stimulus was given every other time that a certain syllable (KAX) appeared on the screen. The reaction was recorded as GSR. The painful stimulus used was arbitrarily set at 10 volts more than the threshold just previously determined for the individual. In the group in which sound instead of pain was used for the unconditioned stimulus, the exact procedure of Welch and Kubis was followed. A door-bell placed just behind the subject's chair was rung loudly at the appropriate instant (i.e., every other time that KAX appeared). Conditioning time was recorded as that number of times at which KAX had appeared when the first of three consecutive GSRs appeared at an unreinforced syllable. At least 30 trials were given for each subject.

The subjects: These were 57 normal adult controls and 24 adult patients from the Pain Clinic of the University of Oregon Medical School (Table I). The controls were either: the relatives of patients, volunteers from the second year class of medical students, those from a class of student nurses, or members of the Laboratory staff. The patients all suffered from organic conditions involving severe, prolonged pain of months' or years' duration which was still present. The patients' ages varied from 21 to 65 , although only three were over 56 , the average age being 42.9. There were 16 females and eight males. Diagnoses were as follows: causalgia-like syndrome, six; low back pain, five; phantom limb pain, four; intra-abdominal pain, three; arthritis, two; chronic cystic pancreatitis, two; and long-standing coronary disease, two. All had been unable to work, and in severe discomfort for months, the primary cause for their disability being pain and not any other symptom coincident to their illness. The existent pain syndrome was present and unabated at the time of the tests, although severe pain was present in only five cases (Nos. 39, 105, 110,135, 151). Seven had no pain (Nos. 79, 102, 104, 106, 111, 119, 148) and the remainder felt mild or moderate pain during the procedure. All patients were ambulatory and were attending the Clinic at the time of the testing. Six were at least partially dependent on morphine derivatives (Nos. $39,68,71,106,110,112$ ). As far as could be ascertained, as previously instructed, none had had any medication for 24 hours preceding the test.

Anxiety factor: An attempt was made to appraise the factor of anxiety in the patients. Every effort was made to make the test situation as little alarming as possible. All subjects were asked if they would be willing to cooperate in a project which was designed to investigate the "reactions of people." It was explained that people differed in this respect and that, although the tests might be no direct help to the present pain of the patient, it might ultimately help in understanding his condition and those of others in like difficulties. Each subject was then assured that the procedure would take only about half an hour; that it would not "hurt" and no needles would be used; that no test given would "bother" the subject; and that our method was to use the amount of sweating to measure various reactions.
Both controls and patients were relaxed and co-operative at the time of the test. One subject (No. 79) expressed the idea after the tests that "waiting for those words" had made him feel as though he might be going to have an attack (coronary). Two (Nos. 39, 50) were slightly resentful at being put through the procedure, although they had agreed previously to co-operate. Without knowledge of the test results a "most anxious" and "least anxious" group were selected by three individuals who knew the patients well (the doctor in the Clinic, the doctor guiding the experimental procedure and the psychologist) and these ratings were later examined in relation to the tests. All but two of the patients-the two with chronic arthritis-had been described earlier as either having "purely functional" pain or at least a "strong functional element" by the doctors who had sent them to the Pain Clinic. There were eight of the 24 who were definitely of the over-reactive type, chronic habitués of all clinics, whose focus of attention in life was undoubtedly upon their somatic ills (Nos. 38, 39, 55, 96, 101, 109, 111, 112). However, all 24 patients had true focal pain of organic origin.

\section{Test procedure}

The subject was seated in a comfortable chair facing the projection screen and turned away from the investigator and the recording apparatus. The movie projector was above and behind the chair. The directions given were almost identical for each individual throughout the procedure, the author and one other person being present at each test. Tests were always given in the same sequence.

Test One: The subject was told that this test was "just to try out the apparatus." $\mathrm{He}$ was then asked to think of "any number between one and 10 inclusive" and to think about that single number persistently without replying to the investigator's queries, the machine being turned on during the procedure. He was asked, "Is it three?," "Is it nine?," etc., etc. Each number was asked twice, the sequence being random but invariable since it was read from a card. The GSR record was then examined. In the event that a marked GSR swing had appeared for a single number the subject was told what his choice had been. This test served several purposes-to be sure that the system was working; to allow calibration of the GSR swing; and to reassure the subject who was usually amused or a little incredulous, according to his sophistication in such matters.

Test Two: Pain threshold. Starting well below threshold, graded stimuli were given from the Goodwin Stimulator. Each stimulus was given twice at about one second's interval and the subject became used to the click of the switch whether a stimulus was felt or not. No GSR recording was made at this time, but the subjective impression of the patient was written on the record. At a given point, as the threshold was increased, the subject felt a touch or tickle (" $T$ " in Table I). He was then told that the stimulus would be gradually increased "like this" and that at a given point he would feel a prick or pain instead of a touch, but that it would never be a pain which 
would "make you burst out crying." This threshold for pain ("P" in Table I) was then noted on the record. During this test the stimulus gradient was always increased 10 volts at a time without any variation in the procedure. After the pain threshold had been reached two or three more stimuli were delivered just above, below and at threshold to check its level. The subject was then told that subsequent prick stimuli would never be more intense than those already experienced.

Test Three: For the conditioning test the pain stimulator was set at 10 volts above the pain threshold just reported. "I want you to read out loud the syllables which I am going to throw on the screen in front of you. Never mind how you pronounce them. Some are rather hard to pronounce, but just keep on and on reading them while I turn on the machine and make a record." For this procedure the room was partially darkened. All of the patients and about half of the controls were conditioned to a pain stimulus. The remainder of the controls were conditioned using sound instead of pain for the unconditioned stimulus.

Test Four: At the end of the above, the subject was told that there was just one more brief test in which we would recheck the reactions which had been recorded previously, and that he was merely to sit still with eyes closed for a few minutes while we ran over the previous test. The GSR was then recorded during a sequence of stimuli which started at 10 volts and increased 10 at a time. Five stimuli, three seconds apart, were given at each level, until a GSR response was obtained or until the strength of the stimulus was two units beyond that of the previous pain threshold. Since, with no exceptions, a GSR had been obtained in the just-preceding test at a stimulus level only one unit above threshold for pain, it was felt that two units above must be adequate for the present test. GSR threshold was considered to be that strength of stimulus which produced a GSR in three of the five trials, if that same change was also produced at the level one unit higher. This last condition was found necessary because, in a few instances, and for no obvious reason, a subject might respond three times to a stimulus at a given level but give no further response at several higher levels. When this happened it was thought that there had been fluctuations in the awareness of the subject resulting in re-enforcement of the applied stimulus.

TABLE I

Threshold levels at which touch $(T)$ and pain $(P)$ were felt and at which galvanic skin responses (GSR) and conditioned reflexes $(C R)$ appeared

Pain was used as the conditioning factor in one group of controls and in all patients. Sound was used in the second control group.

\begin{tabular}{|c|c|c|c|c|c|c|c|c|c|c|c|c|c|c|c|c|c|}
\hline \multicolumn{6}{|c|}{ Controls, pain stimuli } & \multicolumn{6}{|c|}{ Controls, sound stimuli } & \multicolumn{6}{|c|}{ Patients } \\
\hline No. & Age & $\mathbf{T}$ & $\mathbf{P}$ & GSR & CR & No. & Age & $\mathbf{T}$ & $\mathbf{P}$ & GSR & CR & No. & Age & $\mathbf{T}$ & $\mathbf{P}$ & GSR & CR \\
\hline $\begin{array}{r}40 \\
45 \\
46 \\
47 \\
59 \\
63 \\
77 \\
81 \\
98 \\
103 \\
107 \\
108 \\
134 \\
137 \\
141 \\
\\
42 \\
43 \\
48 \\
60 \\
62 \\
69 \\
70 \\
72 \\
73 \\
74 \\
75 \\
78 \\
80 \\
132 \\
140\end{array}$ & $\begin{array}{l}25 \\
22 \\
25 \\
21 \\
26 \\
43 \\
27 \\
21 \\
38 \\
28 \\
22 \\
36 \\
37 \\
43 \\
25\end{array}$ & $\begin{array}{l}F e \\
-\bar{Z} \\
\overline{-} \\
20 \\
20 \\
10 \\
20 \\
20 \\
20 \\
20 \\
30 \\
10 \\
20 \\
10 \\
M \\
- \\
30 \\
30 \\
20 \\
40 \\
30 \\
10 \\
40 \\
30 \\
30 \\
40 \\
10 \\
30 \\
50\end{array}$ & $\begin{array}{c}\text { ale } \\
50 \\
70 \\
40 \\
80 \\
70 \\
80 \\
80 \\
90 \\
70 \\
60 \\
60 \\
80 \\
60 \\
60 \\
40 \\
l e \\
80 \\
90 \\
80 \\
80 \\
80 \\
70 \\
90 \\
40 \\
80 \\
70 \\
60 \\
90 \\
60 \\
80 \\
80\end{array}$ & $\begin{array}{l}40 \\
50 \\
50 \\
80 \\
60 \\
60 \\
80 \\
80 \\
80 \\
60 \\
70 \\
60 \\
40 \\
60 \\
50\end{array}$ & $\begin{array}{r}10 \\
10 \\
10 \\
10 \\
12 \\
4 \\
6 \\
9 \\
16 \\
8 \\
4 \\
10 \\
6 \\
6 \\
4 \\
\\
10 \\
10 \\
13 \\
11 \\
6 \\
8 \\
8 \\
14 \\
12 \\
4 \\
19 \\
10 \\
2 \\
18\end{array}$ & $\begin{array}{r}53 \\
54 \\
56 \\
58 \\
67 \\
76 \\
125 \\
126 \\
127 \\
128 \\
129 \\
130 \\
133 \\
138 \\
139 \\
142 \\
143 \\
144 \\
145 \\
146 \\
147 \\
61 \\
65 \\
66 \\
122 \\
123 \\
124\end{array}$ & $\begin{array}{l}23 \\
21 \\
24 \\
37 \\
24 \\
27 \\
20 \\
20 \\
20 \\
20 \\
20 \\
20 \\
20 \\
20 \\
20 \\
20 \\
20 \\
20 \\
25 \\
22 \\
23 \\
20 \\
24 \\
24 \\
25 \\
23 \\
26\end{array}$ & $\begin{array}{c}F e \\
20 \\
30 \\
10 \\
10 \\
20 \\
30 \\
20 \\
20 \\
20 \\
10 \\
10 \\
50 \\
20 \\
10 \\
20 \\
20 \\
10 \\
20 \\
10 \\
20 \\
20 \\
M \\
40 \\
30 \\
30 \\
20 \\
30 \\
10\end{array}$ & $\begin{array}{r}\text { ale } \\
50 \\
50 \\
20 \\
30 \\
70 \\
60 \\
50 \\
50 \\
60 \\
40 \\
60 \\
80 \\
60 \\
50 \\
60 \\
70 \\
60 \\
70 \\
50 \\
70 \\
80 \\
4 l \\
90 \\
50 \\
70 \\
80 \\
80 \\
80\end{array}$ & $\begin{array}{l}20 \\
50 \\
10 \\
40 \\
60 \\
40 \\
50 \\
60 \\
80 \\
50 \\
80 \\
90 \\
50 \\
10 \\
50 \\
60 \\
40 \\
50 \\
50 \\
80 \\
80\end{array}$ & $\begin{array}{r}7 \\
9 \\
7 \\
8 \\
12 \\
2 \\
12 \\
14 \\
4 \\
8 \\
10 \\
8 \\
12 \\
13 \\
12 \\
6 \\
8 \\
10 \\
8 \\
2 \\
4 \\
18 \\
26 \\
6 \\
8 \\
19\end{array}$ & $\begin{array}{r}35 \\
38 \\
39 \\
55 \\
57 \\
90 \\
96 \\
102 \\
104 \\
109 \\
110 \\
111 \\
112 \\
119 \\
121 \\
151 \\
\\
68 \\
71 \\
79 \\
101 \\
105 \\
106 \\
135 \\
148\end{array}$ & $\begin{array}{l}37 \\
28 \\
32 \\
38 \\
35 \\
50 \\
27 \\
30 \\
21 \\
32 \\
56 \\
56 \\
56 \\
38 \\
41 \\
50 \\
\\
55 \\
65 \\
43 \\
40 \\
43 \\
60 \\
45 \\
52\end{array}$ & $\begin{array}{c}\frac{F e}{20} \\
\frac{70}{10} \\
20 \\
20 \\
30 \\
20 \\
20 \\
30 \\
30 \\
30 \\
30 \\
10 \\
10 \\
N \\
20 \\
20 \\
20 \\
20 \\
50 \\
20 \\
40 \\
10\end{array}$ & $\begin{array}{c}\text { zale } \\
40 \\
60 \\
80 \\
90 \\
40 \\
50 \\
50 \\
60 \\
80 \\
60 \\
70 \\
70 \\
80 \\
70 \\
40 \\
30 \\
\text { ale } \\
60 \\
90 \\
50 \\
80 \\
80 \\
70 \\
90 \\
50\end{array}$ & $\begin{array}{l}\overline{20} \\
\overline{-} \\
\overline{-} \\
50 \\
80 \\
60 \\
80 \\
30 \\
80 \\
50 \\
80 \\
80 \\
40 \\
40\end{array}$ & $\begin{array}{r}5 \\
8 \\
10 \\
5 \\
2 \\
10 \\
14 \\
14 \\
14 \\
6 \\
4 \\
2 \\
9 \\
4 \\
\end{array}$ \\
\hline
\end{tabular}


DATA

In Test One, the number secretly selected by the subject was detected from the GSR in $80 \%$ of the controls and $65 \%$ of the patients. This test served chiefly as an indicator of the effectiveness of the test circuit and of the type of reaction which the subject gave. On examining the records later, the ease of detection of the number was listed as either "very clear," "clear," "fair," or "missed." These reactions were sometimes used later in comparing the types of GSR shown by the subject to the pain stimulus and to the conditioning stimulus. It is of interest that there was less consistency in the responses of the patients than in those of the controls, since this variability appeared in some of the later tests of the patients also, but in spite of other attempts at correlation with type of clinical diagnosis or state of anxiety, nothing more objective emerged from this procedure.

In Test Two the thresholds for touch and pain as detected subjectively were noted. As can be seen from Table I, threshold for the perception of touch varied between 10 and 50 volts. There were eight subjects on whom touch thresholds were not recorded as it was not recognized in these earlier tests that it might be significant. There were 18 (14 males) of the remaining 51 controls and eight
TABLE II

Mean thresholds for touch $(T)$, pain $(P)$, galvanic skin response $(G S R)$ and conditioning times $(C R)$

\begin{tabular}{l|c|c|c|r}
\hline \hline & \multicolumn{4}{|c}{ Mean thresholds } \\
\cline { 2 - 5 } & $\mathrm{T}$ & $\mathrm{P}$ & $\mathrm{GSR}$ & $\mathrm{CR}$ \\
\hline Patients, male & 25.00 & 71.25 & 62.00 & 10.42 \\
Patients, female & 22.86 & 60.63 & 57.50 & 7.64 \\
Controls, male (pain) & & & & 10.38 \\
Controls, female (pain) & & & & 8.33 \\
Controls, male (sound) & & & & 13.50 \\
Controls, female (sound) & & & & 8.00 \\
Total controls, male & 28.33 & 75.17 & 66.33 & 11.94 \\
Total controls, female & 18.62 & 63.33 & 56.82 & 8.47 \\
\hline
\end{tabular}

(two males) of the 22 patients with touch thresholds above 20 .

Pain threshold varied between 30 and 90 volts in the controls and between 30 and 90 volts in the patients. Table II shows the means for the two groups and for the sexes. It will be seen that the thresholds for touch and pain are higher in the males than in the females in both patients and control group (Figure 1). But when the 24 patients were matched for age and sex with 24 of the controls (Figure 2), no difference between the two became apparent.

The results of Test Three, conditioning to pain and sound, are shown in Figure 3. With either stimulus there was no significant difference be-

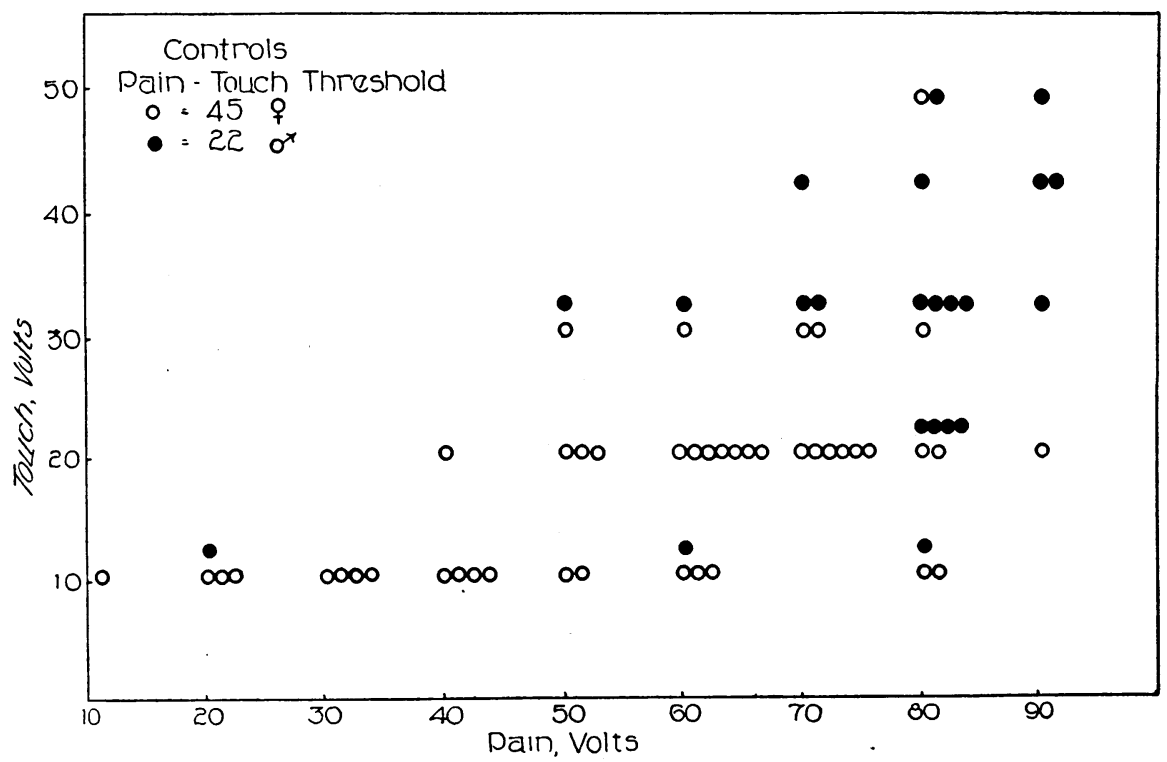

Fig, 1. Pain and Touch Threshold of Control Groups Showing a Higher Threshold for Males than for Females 


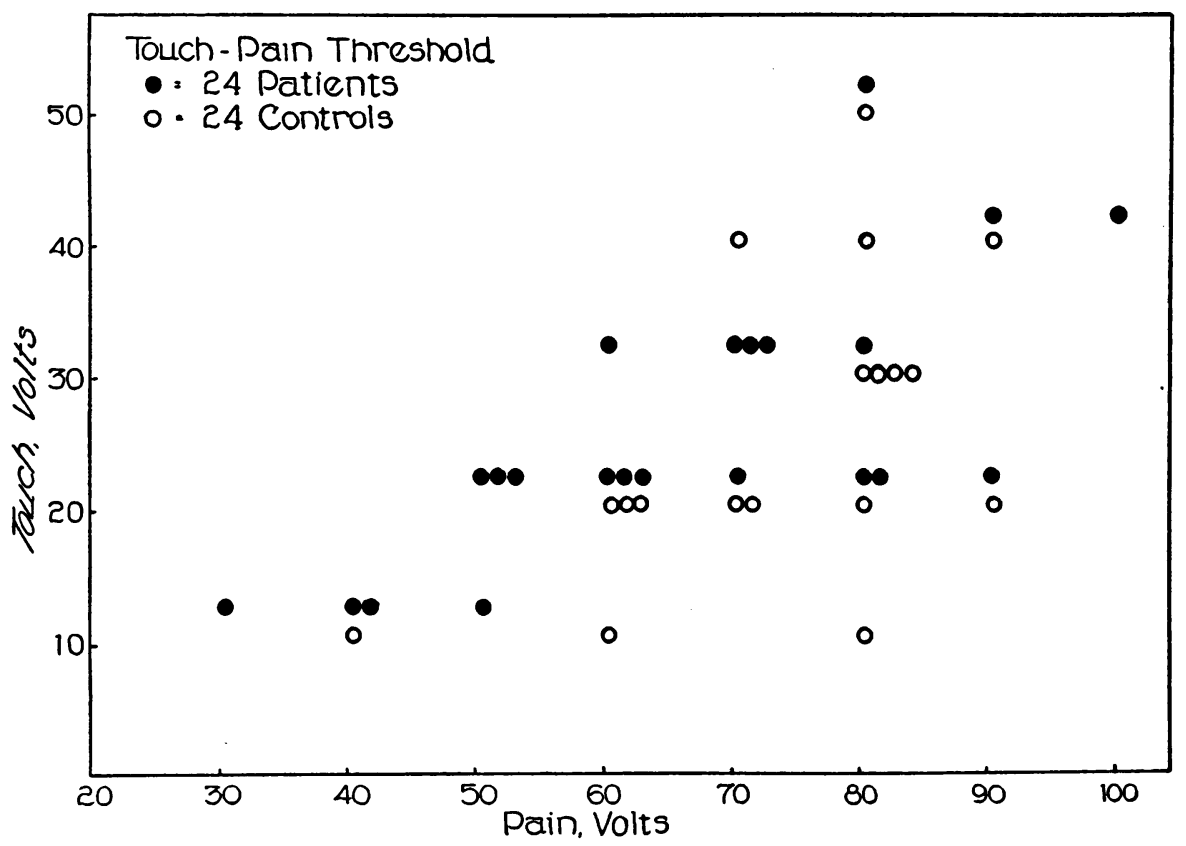

Fig. 2. Pain Thresholds for 24 Pattents and 24 Controls Showing no Significant Differences

Here, the age and sex of the 24 patients has been matched by that of the selected controls.

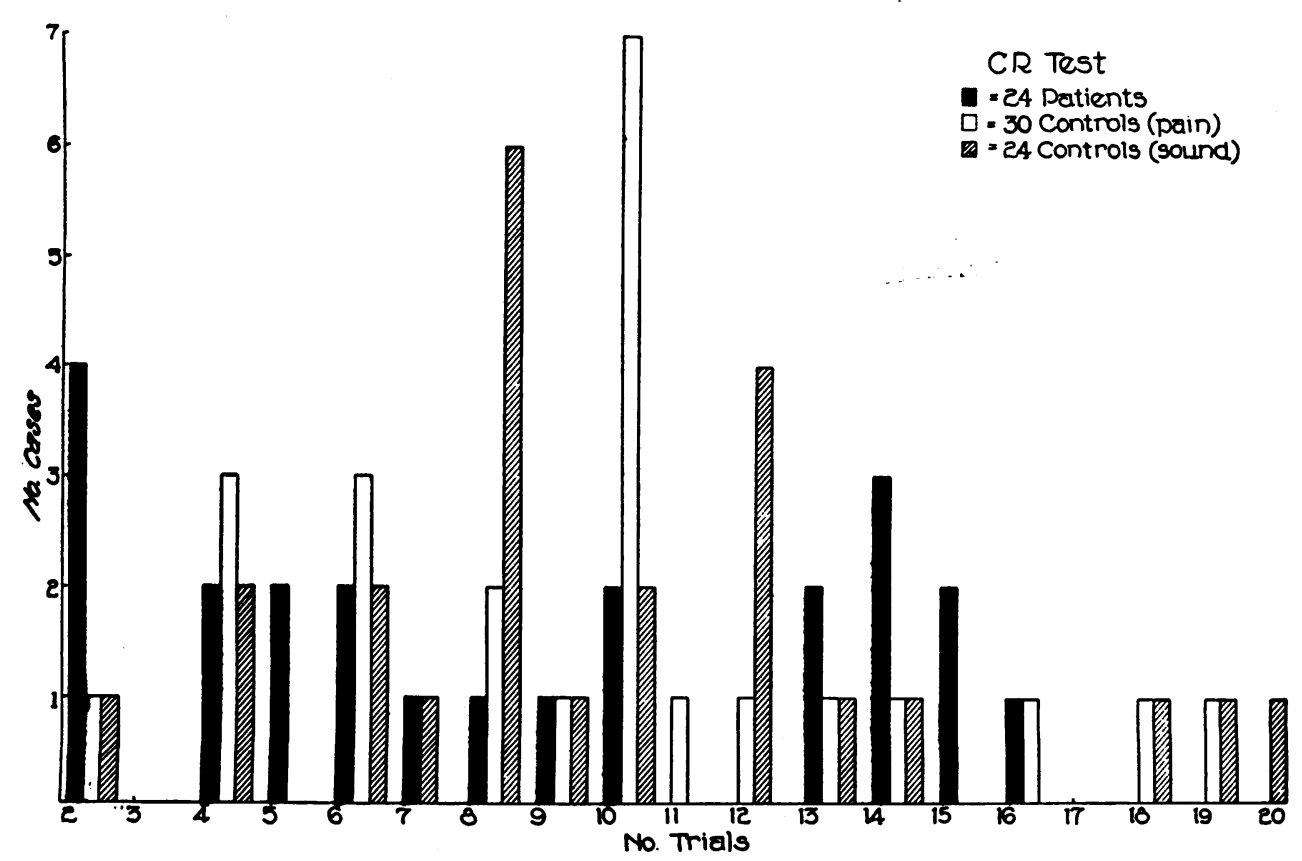

Fig. 3. Conditioning Time and Number of Trials of 24 Patients and 30 Controls Using Pain as the Conditioning Stimulus and for 24 Controls Using a Sound Stimulus No significant differences appear. 
tween controls and patients. When the controls, using the pain stimulus as originally planned, showed a much lower level for conditioning rate than had been previously reported as normal (4), it was thought that this might be due to the fact that a pain stimulus was more anxiety-inducing than a sound stimulus. A second control group was therefore selected, using sound as in the original procedure, instead of pain (4). There was no difference in conditioning time between the three groups, although a slight sex difference in all three appeared. Since the conditioning time varied only between two and 26 , and since there were only seven of the 81 subjects who conditioned in more than 15 trials, differences would have had to be very large and clear-cut to be significant. These did not appear.

Welch and Kubis divided their patients according to whether they conditioned below six trials or above 14, and found positive correlation between such groupings and the clinically rated amount of anxiety. When the 24 patients of our group were classified according to their clinical anxiety as described above, eight patients were thought by all observers to be "most anxious" and eight "least anxious." The average conditioning time of the "anxious" group was 6.9 and that of the less anxious 13:0. Both these means, however, are below the average for normal as designated by Welch and Kubis.

Three patients and two controls gave results which could not be interpreted. Of these, two patients and one control reacted so strongly to every syllable that no conditioning could be measured. The other two never developed a conditioned reaction although responses to unconditioned stimuli were present.

The thresholds for the GSR as recorded in Test Four showed no significant difference between the controls and patients although the responses of the patients were more widely scattered than were those of the controls. Although pain threshold for males was higher than for females, this difference was less great in the reaction to pain as indicated by the GSR threshold. As might be expected by the data of Furer and Hardy (3), the GSR was much more variable than was the pain threshold. Seven of the 24 patients, but none of the controls, had reactions which were so con- fused that no threshold could be determined. Five of these had no GSR to any stimulus given during the fourth test although they had shown definite reactions to smaller stimuli during the number and conditioning tests. They must, therefore, have accommodated to the GSR during the previous testing. The other two had such marked over-reactions both during the GSR and the conditioning tests that no threshold or conditioning time could be determined. No correlation was found between these unusual reactions and anxiety ; presence or absence of pain; or clinical diagnosis. But the conditioning time of the seven whose GSRs were undeterminable in Test Four averaged 6.8 which is relatively fast as compared to the average for all patients.

There was also slightly more variability or irregularity in pain GSR relationships of the patients than of the controls. In the controls the usual GSR threshold was either equal or slightly below the pain threshold, but this relationship was much more variable among the patients. Similarly, extraneous GSR responses appeared more often and were more marked in the patients' records.

\section{DISCUSSION}

The original purpose of this investigation was to discover whether there were any significant differences between the responses to painful stimuli of patients who had endured long and severe pain and controls who had not. Most of the findings are negative. There is not, as shown by these tests chosen to measure various qualities related to pain, any significant difference in threshold, reaction time or conditioning to pain, between patients and controls. The data are reasonably consistent and, therefore, assumed valid. They expose a number of interesting points in relation to our present knowledge of the physiology of pain.

Thresholds for touch and pain were detected consistently by the subjects and it was felt that the electrical stimulus used was the one of choice for this purpose both because the procedure was greatly shortened when compared to that necessary in using a heat stimulus and because the pain threshold so detected had been shown to be fairly consistent in previous tests repeated on the Laboratory staff. 
In these tests the threshold for both touch and pain was higher in males than in females. There are several possible reasons for this, but no completely satisfactory explanation. It may be that, in the males, the skin of the forearm is thicker. The oldest and thickest-skinned males were compared, therefore, with the youngest, and probably most tender-skinned but no difference in threshold was apparent. Moreover, if the skin thickness were important, it should also have affected the pain threshold for heat radiation, but there is no such sex difference reported by those who have used the latter test. Another responsible factor might be the mores of the present time. Men are not supposed to cry out to pain as quickly and easily as are women. Whether this could influence our data is difficult to decide, and again, if these habits were responsible they might have affected the data of Wolff and his associates also. However, their tests were carried out largely on highly trained and sophisticated controls which might have influenced this special point. Furthermore, the fact that our GSR levels for the two sexes were more nearly alike than were touch or pain thresholds, might also indicate habit differences, for the GSR is less "voluntary" than is the subjective response to stimuli.

The GSR, like the pain threshold, produced nothing of interest which might differentiate patient from control except that, in general, reactions were more variable and less clearly defined in the patient group. Nothing could be found which related clinical features such as anxiety or a functional overlay, to any type of reaction. The heightened variability in the patients' test may be due to one of several factors. These patients, having only their pain in common were a more heterogeneous lot than were the controls, since the patients had greater range in age and since their educational and economic backgrounds were more varied. In a number of cases it was felt by the observers that the attention of the patients was less well focused than that of the control group. This was, in some instances, due to the fact that the patient was relatively uncomfortable because of his existent pain. In others, the greater drive and interest of the patient in the tests appeared to make his attention wander away from the particular focus-to the observer, the noise of the machine, etc. Anxiety, in this immediate sense, as set up in relation to the tests was thought to be a deterrent from efficient performance rather than a stimulus to it.

The fact that both control groups had mean levels for conditioning time which were about equal to that of the patients is not necessarily surprising, but the fact that the control groups showed levels which would place them, according to the categories of Welch and Kubis (4), in a group having high anxiety status is difficult to explain and, in itself, invalidates any conclusions which might be drawn from the low conditioning time of the patients. No explanation has been found for this difference between our controls and the normals previously reported. Since the author had previously worked with Dr. Welch, it is probable that the experimental set-up in the present investigation was as nearly like his as possible. As there was no difference between conditioning time of those responding to pain stimuli and to sound, the unconditioned stimulus could not be responsible. It was originally thought that the subjects selected were as nearly like those of Dr. Welch as possible, but it is conceivable that the tension and anxiety factor in a group of graduate students in nursing or medical schools is higher in test situations than that of the more sophisticated psychology classes of Hunter College. The processes underlying this difference are not easy to detect. The fact that the project had to do with pain may have had some influence. The personality and performance of the observer may also have been effective. All that is shown, apparently, by the test is that the patient and control groups are similar in their performances, and that there are other factors as well as those of anxiety, originally described as relevant, which have not yet been evaluated and which must play a part in the interpretation of the test.

In concluding, it should be noted that the data obtained from psychological tests on these same patients, which are to be published elsewhere, showed similar findings to the above in that the mental status and personality types of the patients showed no uniformity of trend which could be related either to functional or anxiety factors or to the behavioral reactions to pain. This group of 24 patients, in spite of the fact that most of them 
had previously been categorized as being overreactive or having functional elements to their pain reacted on the whole like any other group of individuals except that in addition to their many and varied and normal characteristics, they had also their severe and chronic pain.

\section{CONCLUSIONS}

1. Comparisons have been made between the responses to painful stimuli of normal control subjects and of patients having chronic clinical conditions involving severe, long-standing pain.

2. The thresholds for touch and pain perception were recorded. No significant difference appeared between the levels of the controls and those of the patients.

3. Reaction to pain, as indicated by the galvanic skin response, was also similar in the control and in the patient groups. As was to be expected from the work of previous authors, reaction to pain was more variable and less constant than was pain threshold.

4. Conditioning to painful and to auditory stimuli proved to be alike in the two groups.

5. Significant differences in touch and pain . threshold and slight differences in GSR threshold and in conditioning time, appeared between males and females irrespective of whether they were in the control or patient groups.
6. In spite of the fact that all patients had signs of focal organic pain-inducing lesions, about $80 \%$ had been previously diagnosed as "functional." But there was no evidence that anxiety and tension, as measured by the present tests, differed in the patient and control groups.

7. The only difference observed in test results was that the responses of the patients were more varied and less clear-cut than those of the controls. This variability could not be related to any known factor within the groups unless it was that the attention of the patients was less well directed and maintained.

\section{REFERENCES}

1. Hardy, J. D., Wolff, H. G., and Goodell, H., The pain threshold in man. Am. J. Psychiat., 1943, 99, 744.

2. Hardy, J. D., Wolff, H. G., and Goodell, H., Studies on pain: discrimination of differences in intensity of a pain stimulus as a basis of a scale of pain intensity. J. Clin. Invest., 1947, 26, 1152.

3. Furer, M., and Hardy, J. D., The reaction to pain as determined by the galvanic skin response. A. Res. Nerv. \& Ment. Dis., Proc., 1949, 29, 72.

4. Welch, L., and Kubis, J., Conditioned PGR (psychogalvanic response) in states of pathological anxiety. J. Nerv. \& Ment. Dis., 1947, 105, 372.

5. Redlich, F. C., Organic and hysterical anesthesia. A method of differential diagnosis with the aid of the galvanic skin response. Am. J. Psychiat., 1945, 102, 318 . 